\title{
More Aggressive Cancer Behaviour in Thyroid Cancer Patients in the Post-COVID-19 Pandemic Era: A Retrospective Study
}

\author{
Hanqing Liu' ${ }^{1, *}$ \\ Ling Zhan ${ }^{1} *$ \\ Liantao Guo' \\ Xizi Yu' \\ Lingrui $\mathrm{Li}^{\prime}$ \\ Hongfang Feng ${ }^{2}$ \\ Dan Yang ${ }^{3}$ \\ Zhiliang $\mathrm{Xu}{ }^{\prime}$ \\ Yi Tu' \\ Chuang Chen' \\ Shengrong Sun (iD)
}

'Department of Thyroid and Breast Surgery, Renmin Hospital of Wuhan University, Wuhan, 430060, Hubei, People's Republic of China; ${ }^{2}$ Department of Thyroid and Breast Surgery, Huangshi Central Hospital of Edong Healthcare Group, Hubei Polytechnic University, Huangshi, 435000, Hubei, People's Republic of China; ${ }^{3}$ Department of Cardiology, Renmin Hospital of Wuhan University, Wuhan, 430060, Hubei, People's Republic of China

*These authors contributed equally to this work

\begin{abstract}
Purpose: Many thyroid cancer patients have suffered from treatment delays caused by the coronavirus disease 2019 pandemic. Although there have been many reviews, recommendations, or clinical experiences, clinical evidence that evaluates patient disease status is lacking. The aim of our research was to evaluate thyroid cancer behaviour in the post-COVID-19 era. Patients and Methods: A retrospective study was conducted and thyroid cancer patient data from February 1, 2017 to September 15, 2020 were pooled for analysis. The demographic, ultrasound and pathological data of the pre- and post-COVID-19 groups were compared. Lymph node metastases, tumour size, extrathyroidal extension, and multifocality were compared year-by-year to evaluate annual changes in patient characteristics. Regression analyses were adopted to reveal cancer behaviour along with the admission date interval and to reveal risk factors for lymph node metastasis. Patient ultrasound data were compared before and after the lockdown to assess tumour progression. The outcomes of delays in treatment $\leq 180$ days were then studied.

Results: The post-lockdown patients were more likely to have multiple lesions $(31.2 \%$ vs $36.5 \%, p=0.040)$, extrathyroidal extension $(65.5 \%$ vs $72.2 \%, p=0.011)$ and lymph node metastases $(37.7 \%$ vs $45.0 \%, p=0.007)$, while tumour size remained stable $(1.01 \mathrm{~cm}$ vs. $1.02 \mathrm{~cm}, \mathrm{p}=0.758)$. The lymph node metastasis rate increased by year $(p<0.001)$. The tumour size correlated negatively with the post-lockdown admission date $(p=0.002)$. No significant difference in tumour size, multifocality or lymph node metastasis on ultrasound was revealed between the pre- and post-lockdown group. No significant difference in tumour size, multifocality, extrathyroidal extension or lymph node metastasis was revealed among patients with a delayed treatment time $\leq 180$ days.
\end{abstract}

Conclusion: Patients with a COVID-19-induced treatment delay had more aggressive cancer behaviour. Rebound medical visits and annually increasing aggressiveness may be potential reasons for this observation, as individual patient tumour did not progress during the delay.

Keywords: treatment delay, lockdown, rebound medical visit, tumor behavior

\section{Introduction}

Since the first patient was discovered in Wuhan, China, in December 2019, the coronavirus disease 2019 (COVID-19) pandemic has spread around the world and has led to over 220 million infections and approximately 4.6 million deaths in 220 countries worldwide. ${ }^{1}$ To minimize the risk of interpersonal transmission, many medical institutions have adopted protection strategies, such as teleconsultation, mandatory tests for COVID-19 antibodies and nucleic acids, the wearing of masks or other personal 
protection equipment, and admission restrictions. ${ }^{2-5}$ These methods have shown protective effects in the fight against COVID-19. Despite the beneficial effects, these protective approaches may have a negative impact on the clinical management of patients.

Some cancer patients have been advised to reduce their visits to medical institutions to avoid nosocomial infections. As a consequence, a large percentage $(38.7 \%$ to $59.0 \%$ ) of cancer patients were reported to have experienced a COVID-19-induced treatment delay. ${ }^{6-8}$ Severe anxiety and depression were observed among those patients. ${ }^{9}$ Although a three-month delay in cancer treatment was deemed tolerable in some studies and recommendations, ${ }^{10,11}$ such advice must be adopted with great caution. Obviously, cancers originating from different tissues need to be handled according to their severity and aggressiveness. Patients with aggressive cancers, such as non-small-cell lung cancer, acute myeloid leukaemia, or melanoma, will have a much poorer prognosis if their active interventions are postponed. ${ }^{12-14}$ In contrast, those with indolent tumours, including most prostate cancers or differentiated thyroid carcinomas, may tolerate a rather long-term delay without cancer disease progression. ${ }^{15,16}$ The above current clinical studies have focused on the poor prognoses of high-risk cancers, while clinical evidence is lacking in low-risk cancers.

Thyroid carcinoma is the most frequently diagnosed cancer of the endocrine system, and its incidence has increased threefold over the past 2 decades. ${ }^{17}$ Although most studies have attributed the increasing incidence to overdiagnosis, ${ }^{18,19}$ some other scholars have debated whether there is a concurrent true increase in disease occurrence. Evidence supporting this hypothesis includes an increasing mortality rate (by an average of $2.9 \%$ per year) and increasing numbers of large tumours, extrathyroidal extension and cervical metastases. ${ }^{20,21}$ A hypothesis may thus be deduced that there has been a true increase in severity over the years. In addition, patient worry is another factor to be considered. A previous study supported that thyroid cancer patients with more aggressive cancer behaviour tended to be more worried. ${ }^{22}$ Those worried patients may have been more active in seeking for medical care, ${ }^{23,24}$ which may have led to a pseudo-increase in the perceived severity of the disease after the lockdown. Moreover, although thyroid cancers are indolent in most cases, there is a possibility that some patients have suffered tumour progression during the pandemic-induced lockdown. According to guideline, cervical ultrasound is the first choice in monitoring tumour progression, ${ }^{25}$ and some patients underwent cervical ultrasound examinations both before and after the pandemic-induced lockdown.

During the COVID-19 pandemic, Wuhan implemented a lockdown policy from January 23rd to April 8th, 2020. Some oncology departments were partly closed for 3 to 6 months (varying by hospital) when almost all thyroid cancer patients were advised to stay at home to reduce social contact. ${ }^{26}$ This study took the rare and valuable opportunity presented by the lockdown to evaluate the natural development of thyroid cancer and to investigate the change in cancer behaviour in the pre- and postlockdown era and its potential reasons.

\section{Materials and Methods Study Design and Participants}

This single-centre, retrospective, cohort study was conducted at Renmin Hospital of Wuhan University. Data were collected from February 1st, 2017 to September 15 th, 2020. All patients $\geq 18$ years old, and admitted to Renmin Hospital of Wuhan University with any histological types of thyroid cancer as evidenced by postsurgical pathological sections were included in this study. Included patients had to have complete pathological data. This was a four-step study. First, the demographic and postsurgical pathological characteristics were compared between the pre- and post-lockdown groups to assess whether cancer behaviour after the lockdown was more aggressive. If the hypothesis was confirmed, the remaining three steps were designed to evaluate the potential reasons. Divided according to the year of their admission, patients' characteristics were then compared to evaluate the annual change in the thyroid cancer patient spectrum. Afterwards, patients admitted after the lockdown were analyzed as a subgroup and their characteristics, along with their admission dates, were evaluated to study the hypothesis of an increase in cases being due to rebound medical visits. Finally, those patients, who were scheduled for surgery but failed to be admitted before the lockdown and then received surgery after the reopening, were considered separately to study the change in individual tumour behaviour among this subset. All patients included in our study were scheduled for surgery based on ultrasound or/and fine-needle aspiration biopsy findings. Medical equipment and criteria remained unchanged over the four years. This study was approved by the Independent Ethics Committee (IEC) of Renmin Hospital 
of Wuhan University (number: WDRY2020-K231) and was conducted in compliance with the Declaration of Helsinki. Patients were anonymized and their data confidentiality was protected. Since this was a retrospective observational study with no intervention, the requirement for informed consent was waived according to the IEC guidelines.

\section{Data Collection and Definition}

Six independent investigators collected the data in standardized case report forms and cross-checked them with each other. The patients' demographic characteristics, clinical presentations, pathological data, sonographic data, laboratory values, treatments, and postsurgical conditions were obtained from the Hospital Information System. Comorbidities included hypertension, diabetes mellitus, cerebrovascular diseases and other chronic diseases. Other concomitant thyroid diseases were excluded from comorbidities. The pre-COVID-19 ultrasound data were collected based on the last ultrasound before the pandemic. Lesions were evaluated in accordance with the Thyroid Imaging, Reporting and Data System (TI-RADS) of the American College of Radiology. ${ }^{27}$ The delay time was defined as the interval between the date of the last prepandemic ultrasound and the date of the pre-surgical ultrasound. The admission date interval (ADI) was defined as the interval between the patient admission date and April 8th, 2020, which was the last day of lockdown. The data were then input into a database for further analysis.

\section{Statistical Analysis}

Patient numbers and percentages were calculated for categorized data. Continuous data are presented as the means (SDs) or medians (quantiles). The comparisons of clinical characteristics between different cohorts were conducted using the Pearson chi-square test or McNemar test for categorized data, the $t$-test or analysis of variance for continuous data, and the Mann-Whitney $U$-test or Wilcoxon's signed-rank test for ordinal data. Logistic regression analysis was employed to analyse the risk factors for lymph node metastasis. Logistic and linear regression analyses were also adopted to evaluate the correlation between admission date interval and pathological characteristics. A $p$ value $<0.05$ was considered statistically significant. The statistical analysis was performed with SPSS version 23.0 (SPSS Inc, Armonk, NY).

\section{Results}

\section{Demographic and Clinical Characteristics}

From February 1, 2017 to September 15, 2020, a total of 3216 thyroid cancer patients with pathological evidence of disease were included in our study (Figure 1). Patients were divided into the pre-lockdown and post-lockdown groups according to their surgery dates (Supplementary Figure 1). Female patients accounted for $75.5 \%$ of all in-hospital patients. The mean and median ages were 45.9 and 47 years, respectively, with no significant difference between the pre- and post-lockdown groups. Comorbidities were more common before the pandemic $(p=0.001)$. Hashimoto's thyroiditis was discovered in $10.0 \%$ of patients with no significant difference observed between the two groups $(p=0.061)$. Among the patients, $13.7 \%$ reported that at least one of their family members had cancer or a personal history of another malignancy (Table 1).

\section{The Comparison of Pathology Before and After the Lockdown}

The dominant histological type was papillary thyroid carcinoma, which accounted for $98.7 \%$ of the total number of patients. Follicular $(0.9 \%)$ and medullary $(0.5 \%)$ carcinomas accounted for the remaining proportion, while anaplastic carcinoma was discovered in only six patients. A small number of patients had more than one type of cancer. Papillary thyroid microcarcinomas (PTMC) were found in $61.5 \%$ of all patients. The average maximum size of tumour was $1.01 \mathrm{~cm}$ in the pre-lockdown group and $1.02 \mathrm{~cm}$ in the post-lockdown group $(p=0.758)$. Patients after the lockdown tended to have a greater number of multiple lesions

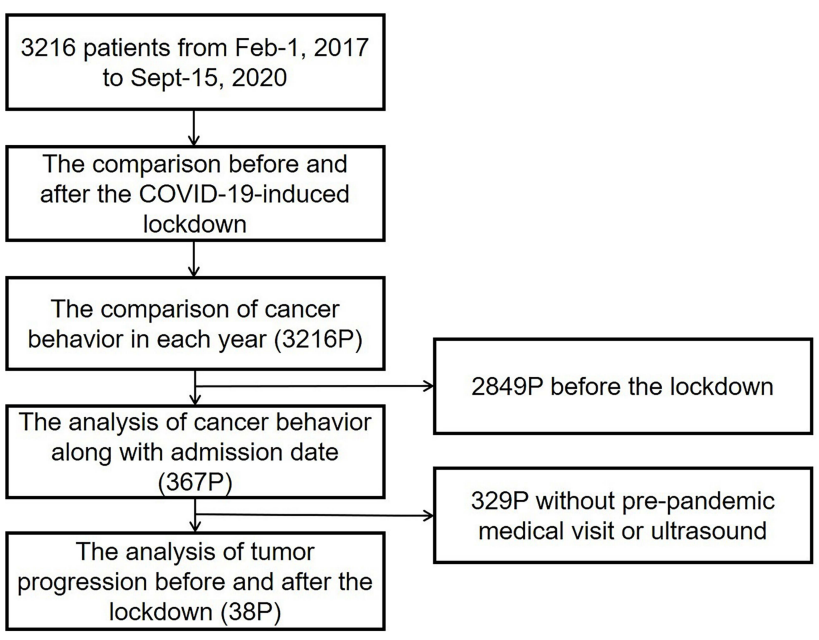

Figure I The flow chart of the study. P is the abbreviation of "cases". 
Table I Cohort Clinical Characteristics of 3216 Patients with Thyroid Cancer Before and After the COVID-19-Induced Lockdown ${ }^{\mathrm{a}}$

\begin{tabular}{|c|c|c|c|c|}
\hline Variable & Total & Pre-Lockdown & Post-Lockdown & $P$ value \\
\hline Sex, female (\%) & $2427(75.5)$ & $2137(75.0)$ & $290(79.2)$ & 0.079 \\
\hline \multicolumn{5}{|l|}{ Age } \\
\hline Mean (SD) & $45.9(11.9)$ & $46.0(11.9)$ & $45.3(11.5)$ & 0.275 \\
\hline Median (IQR) & $47(37,54)$ & $47(37,54)$ & $46(36,53)$ & 0.381 \\
\hline$\leq 50 y(\%)$ & $2009(62.5)$ & $1776(62.3)$ & $233(63.5)$ & 0.668 \\
\hline Comorbidities (\%) & $836(26.0)$ & $768(27.0)$ & $68(18.5)$ & $0.001 * *$ \\
\hline Hashimoto's thyroiditis (\%) & $323(10.0)$ & $276(9.7)$ & $47(12.8)$ & 0.061 \\
\hline Familial or personal history of cancer (\%) & $442(13.7)$ & $400(14.0)$ & $42(11.4)$ & 0.174 \\
\hline \multicolumn{5}{|l|}{ Histological type and subtype (\%) } \\
\hline Papillary & 3174 (98.7) & 2811 (98.7) & $363(98.9)$ & 0.699 \\
\hline PTMC & $1978(61.5)$ & $1750(61.4)$ & $228(62.1)$ & 0.795 \\
\hline Follicular & $30(0.9)$ & $27(0.9)$ & $3(0.8)$ & 0.807 \\
\hline Medullary & $16(0.5)$ & $16(0.6)$ & $0(0.0)$ & 0.150 \\
\hline \multicolumn{5}{|l|}{ Postoperative pathological information } \\
\hline Maximum size (SD) & $\mathrm{I} .0 \mathrm{I}(0.83)$ & $\mathrm{I} .0 \mathrm{I}(0.83)$ & $1.02(0.84)$ & 0.758 \\
\hline Multifocal lesion (\%) & $1023(31.8)$ & $889(31.2)$ & $134(36.5)$ & $0.040 *$ \\
\hline Extrathyroidal extension (\%) & $2132(66.3)$ & $1867(65.5)$ & $265(72.2)$ & $0.011 *$ \\
\hline Lymph node metastasis $^{c}$ & $1240(38.6)$ & $1075(37.7)$ & $165(45.0)$ & $0.007 * *$ \\
\hline CLNM (\%) & $1199(37.3)$ & $1038(36.4)$ & $161(43.9)$ & $0.006 * *$ \\
\hline LLNM (\%) & $262(8.1)$ & $222(7.8)$ & $40(10.9)$ & $0.041 *$ \\
\hline CLN positive number (SD) & $3.79(3.60)$ & $3.78(3.60)$ & $3.81(3.60)$ & 0.942 \\
\hline LLN positive number (SD) & $6.20(5.27)$ & $5.97(5.19)$ & $7.45(5.62)$ & 0.102 \\
\hline
\end{tabular}

Notes: Bold font: significant difference. ${ }^{*} p<0.05,{ }^{*} p<0.01 .{ }^{a} \mathrm{~A}$ number of 2849 patients before the lockdown and 367 after the lockdown were included in the analysis. The admission date of included patients ranged from Feb. I, 2017 to Sept. 15, 2020. ' Other concomitant thyroid diseases were excluded from comorbidities. ${ }^{\mathrm{C}} \mathrm{CLN}$ and LLN positive number was calculated in 1200 patients with CLN metastasis and 259 with LLN metastasis, respectively.

Abbreviations: PTMC, papillary thyroid micro carcinoma; CLNM, central lymph node metastasis; LLNM, lateral lymph node metastasis.

than patients before the pandemic $(p=0.040)$. In the postlockdown patient groups, extrathyroidal extension $(p=$ $0.011)$, and lymph node metastases $(p=0.007)$ were more common than in the pre-pandemic patient group. In addition, the central and lateral lymph node positive rates increased in the post-pandemic group (36.4\% vs $43.9 \%$, $p=0.006,7.8 \%$ vs $10.9 \%, p=0.041$ ) (Table 1 and Supplementary Figure 2A and $\underline{B}$ ).

\section{The Comparison of Pathology in Each Year}

A comparison of postoperative pathology was then conducted for each year of the study's duration. Patients were divided into four groups based on their admission year. The main postoperative pathological characteristics, including tumor size, lymph node metastasis (LNM), and extrathyroidal extension (ETE), were compared. The proportion of patients with LNM increased gradually from
$32.2 \%$ in 2017 to $45.0 \%$ in $2020(p<0.001)$. The uprising rate of central LNM was the principal cause $(p<0.001)$ while the rate of patients with lateral LNM remained stable over the same four years $(p=0.150)$. In addition, no significant difference was observed in tumour size $(p=$ $0.194)$, multifocality $(p=0.168)$ or $\operatorname{ETE}(p=0.071)$ (Table 2 and Supplementary Figure 2C-F).

Regression analyses were then applied to evaluate the correlations between LNM and demographic characteristics, ultrasound data and admission year. Age $\leq 50$ years $(\mathrm{OR}=2.272, p<0.001)$, female sex $(\mathrm{OR}=0.596, p<$ $0.001)$, tumour size (OR $=1.391, p<0.001)$, and the TIRADS score $(\mathrm{OR}=1.296, p<0.001)$ showed to have correlations with central LNM. In addition, female sex $(\mathrm{OR}=0.502, p=0.002)$, tumour size $(\mathrm{OR}=1.487, p<$ $0.001)$, and the TI-RADS score $(\mathrm{OR}=1.589, p<0.001)$ were correlated with lateral LNM. Interestingly, the rate of central LNM increased with admission year $(\mathrm{OR}=1.214$, $p<0.001$ ) (Figure 2 and Supplementary Table 1). 
Table 2 Cohort Clinical Characteristics of 3216 Patients with Thyroid Cancer of Every Year ${ }^{\mathrm{a}}$

\begin{tabular}{|c|c|c|c|c|c|}
\hline Variable & 2017 Feb- & 2018 Feb- & 2019 Feb- & 2020 Feb- & $P$ value \\
\hline Sex, female (\%) & $503(74.6)$ & $771(77.1)$ & $863(73.5)$ & $290(79.2)$ & 0.074 \\
\hline \multicolumn{6}{|l|}{ Age } \\
\hline Mean (SD) & $46.4(12.1)$ & $46.2(12.2)$ & $45.5(11.6)$ & $45.2(11.5)$ & 0.198 \\
\hline$\leq 50 y(\%)$ & $396(58.8)$ & $610(60.9)$ & $770(65.6)^{*}$ & $233(63.5)$ & 0.019 \\
\hline Comorbidities (\%) & $200(29.7)$ & $276(27.6)$ & $292(24.9)$ & $68(18.5)^{*}$ & 0.001 \\
\hline Hashimoto's thyroiditis (\%) & $80(11.9)$ & $104(10.4)$ & $92(7.8)^{*}$ & $47(12.8)^{*}$ & 0.007 \\
\hline Familial or personal history of cancer (\%) & $99(14.7)$ & $120(12.0)$ & $18 \mid(\mid 5.4)$ & $42(11.4)$ & 0.057 \\
\hline \multicolumn{6}{|l|}{ Histological type and subtype (\%) } \\
\hline Papillary & $662(98.2)$ & $990(98.9)$ & $1159(98.7)$ & $353(98.9)$ & 0.648 \\
\hline PTMC & $398(59.1)$ & $639(63.8)$ & $713(60.7)$ & $228(62.1)$ & 0.224 \\
\hline Follicular & $10(1.5)$ & $7(0.7)$ & $10(0.8)$ & $3(0.8)$ & 0.401 \\
\hline Medullary & $5(0.7)$ & $5(0.5)$ & $6(0.5)$ & $0(0.0)$ & 0.448 \\
\hline Anaplastic & $\mathrm{I}(0.1)$ & $\mathrm{I}(0.1)$ & $0(0.0)$ & $\mathrm{I}(0.3)$ & 0.460 \\
\hline \multicolumn{6}{|l|}{ Postoperative pathological information } \\
\hline Maximum size (SD) & $1.07(0.94)$ & $\mathrm{I} .00(0.83)$ & $0.98(0.76)$ & $\mathrm{I} .02(0.84)$ & 0.194 \\
\hline Multifocal lesion (\%) & $208(30.9)$ & $323(32.3)$ & $358(30.5)$ & $134(36.5)$ & 0.168 \\
\hline Extrathyroidal extension (\%) & $448(66.5)$ & $648(64.7)$ & 771 (54.7) & $265(72.2)$ & 0.071 \\
\hline Lymph node metastasis (\%) ${ }^{c}$ & $217(32.2)$ & $383(38.3)$ & $475(40.5)^{*}$ & $165(45.0)^{*}$ & $<0.001$ \\
\hline CLNM (\%) & $211(31.3)$ & $368(36.8)$ & $459(39.1)^{*}$ & $161(43.9)^{*}$ & $<0.001$ \\
\hline LLNM (\%) & $48(7.1)$ & $85(8.5)$ & $89(7.6)$ & $40(10.9)$ & 0.150 \\
\hline CLN positive number (SD) & $3.63(3.38)$ & $3.78(3.66)$ & $3.86(3.64)$ & $3.81(3.60)$ & 0.885 \\
\hline LLN positive number (SD) & $5.87(5.28)$ & $6.32(4.33)$ & $5.28(4.43)$ & $7.45(5.62)$ & 0.109 \\
\hline
\end{tabular}

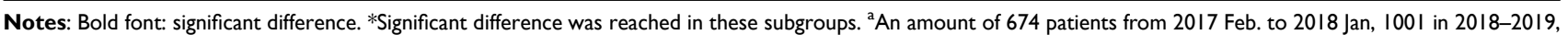
II 74 in 2019-2020 and 367 after the COVID-19-induced lockdown were included in the analysis. The patients in 2020 were limited to Sept. I5, 2020 . ${ }^{b}$ Other concomitant thyroid diseases were excluded from comorbidities. ${ }^{\mathrm{C}} \mathrm{CLN}$ and LLN positive number was calculated in 1200 patients with CLN metastasis and 259 with LLN metastasis, respectively.

Abbreviations: PTMC, papillary thyroid micro carcinoma; CLNM, central lymph node metastasis; LLNM, lateral lymph node metastasis.

\section{Post-Pandemic Admission Date in Predicting Tumour Behaviour}

The correlation between the tumour behaviour and the admission date interval was then evaluated using regression analysis. The data from 367 post-lockdown patients were pooled for the analysis. The admission date interval (ADI) was defined as the time interval between the patient's admission date and April 10, 2020, which was the last day of lockdown. A $p$ value of 0.002 was reached in the correlation between tumour size and the ADI (Supplementary Figure 3). Linear regression analysis showed that the average postoperative pathological tumour diameter decreased by $4 \mathrm{~mm}$ every 100 days. Pathological LNM (OR $=1.000$, $p=0.916$ ), tumour number (slope $=0.000$, $p=0.951)$ and $\operatorname{ETE}(\mathrm{OR}=0.998, p=0.494)$, however, had no correlation with the ADI (Table 3).

\section{Ultrasound Changes Due to a Treatment Delay}

Thirty-eight patients who were scheduled for surgery but who failed to be admitted before the lockdown and received surgery after the reopening were extracted as a subset. These patients received thyroid ultrasound examinations both before and after the COVID-19-induced lockdown. The preCOVID-19 ultrasound was defined as the last ultrasound before the pandemic. Their demographic and clinical characteristics were similar to the total patient sample. The average and median delay time for these patients were 201.3 days and 189 days, respectively (Supplementary Table 2 and Supplementary Figure 4).

More irregular margins were discovered by ultrasound after a long treatment delay $(p=0.012)$. Although there seemed to be some more aggressive features in the postlockdown ultrasound images, no significant difference in 


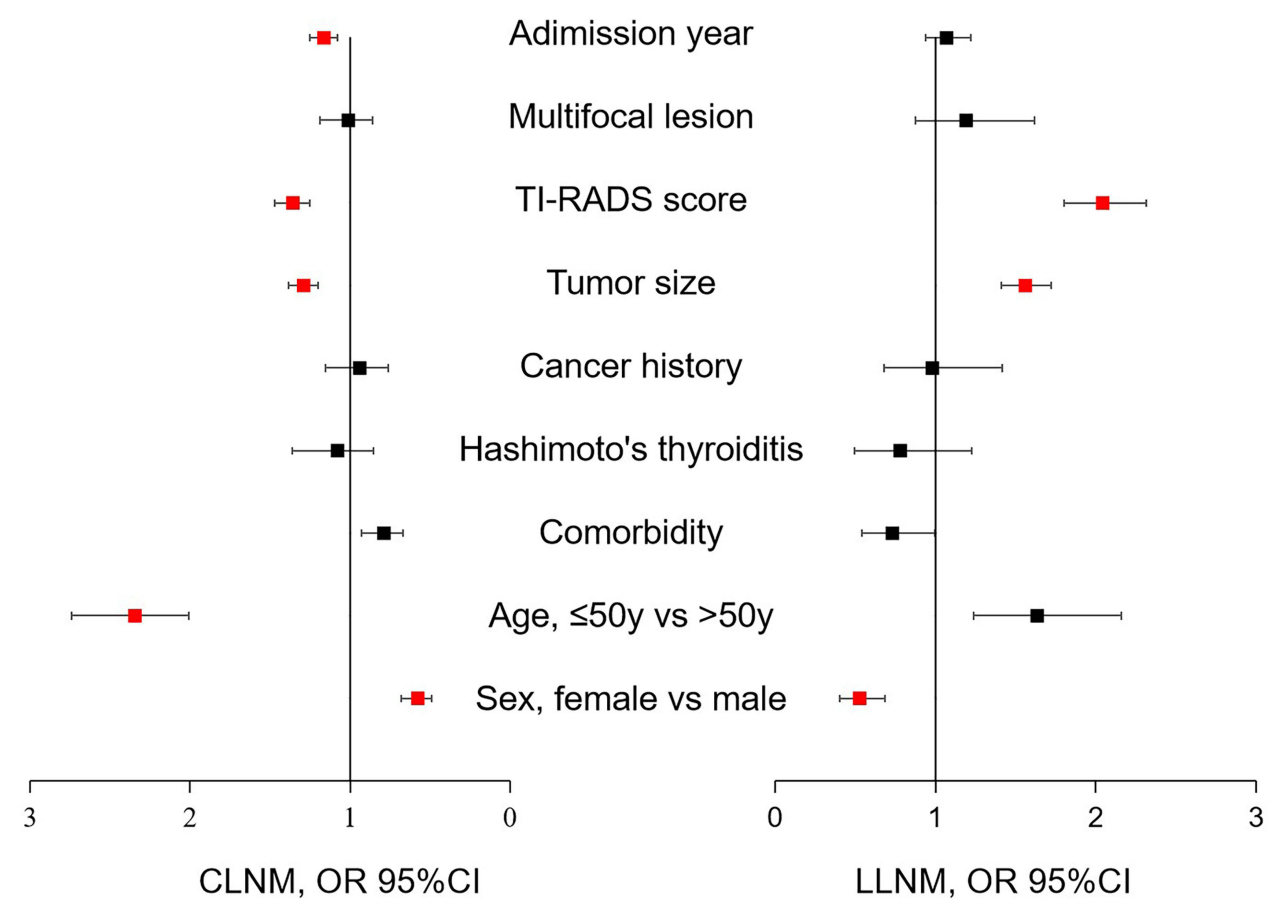

Figure 2 The univariate regression analysis of the correlations between CLNM, LLNM and demographic characteristics, pre-operative ultrasonic data and admission year. Risk factors with red marks had statistical significance in multivariate regression analysis.

Abbreviations: CLNM, lymph node metastasis; LLNM, lateral lymph node metastasis; OR, odd ratios.

the tumour size, aspect ratio, irregular margins, peripheral location, site, microcalcification, multiple lesions, or lymph node metastases was detected across these 38 patients (Table 4).

\section{Outcomes of Treatment Delay in Tumours $\leq 10 \mathrm{~mm}$ and Treatment Delay $\leq 180$ Days in All Patients}

The pre- and post-lockdown ultrasound characteristics were compared across 19 PTMC patients. There was no significant difference in tumour size, aspect ratio, microcalcification, or other ultrasound characteristics. There was no significant difference among the 16 patients whose delay time was less than 180 days (Table 5).

The total patient sample was then divided into two subgroups based on their tumour size. The subgroup with tumours $\leq 10 \mathrm{~mm}$ in diameter showed minimal differences before and after the pandemic-induced lockdown. Those with tumours $>10 \mathrm{~mm}$, however, had more tumour ( 1.67 vs $2.02, p=0.016$ ) and a higher risk of LNM $(72.0 \%$ vs $57.4 \%, p=0.002)$ after the lockdown (Supplementary Table 3 ).

\section{Discussion}

Since its outbreak, the COVID-19 pandemic has been a tremendous challenge to oncology institutions and cancer

Table 3 Regression Analysis of Admission Date in Predicting the Tumor Behavior

\begin{tabular}{|l|l|l|l|}
\hline \multirow{2}{*}{ Dependent Variable } & \multicolumn{3}{|c|}{ Admission Date Interval $^{\mathbf{a}}$} \\
\cline { 2 - 4 } & Effect Size & $\mathbf{9 5 \%} \mathbf{~ I ~}$ & p value \\
\hline Postoperative pathology & & & $\mathbf{0 . 0 0 2 * *}$ \\
Tumor size & -0.004 (Slope, B value) & $-0.006,-0.001$ & 0.916 \\
Lymph node metastasis & 1.000 (OR) & $0.994,1.005$ & 0.951 \\
Tumor number & 0.000 (Slope, B value) & $-0.003,0.004$ & 0.494 \\
Extrathyroidal extension & 0.998 (OR) & $0.992,1.004$ & \\
\hline
\end{tabular}

Notes: Bold font: significant difference. ${ }^{* *} p<0.01$. ${ }^{2}$ The admission date interval was defined as time interval between the patient admission date and April 10, 2020, which was the last day of lockdown. 
Table 4 Comparison of Ultrasound Characteristics in 38 Thyroid Cancer Patients Receiving Ultrasound Examination Before and After the COVID-19-Induced Lockdown

\begin{tabular}{|c|c|c|c|}
\hline Variable & $\begin{array}{l}\text { Pre- } \\
\text { Lockdown }\end{array}$ & $\begin{array}{l}\text { Post- } \\
\text { Lockdown }\end{array}$ & $P$ value \\
\hline Tumor size $(\mathrm{cm}, \mathrm{SD})$ & $1.00(0.92)$ & $1.10(1.18)$ & 0.127 \\
\hline Aspect ratio> I (\%) & $10(26.3)$ & $15(39.5)$ & 0.227 \\
\hline Irregular margin (\%) & $20(52.6)$ & $29(76.3)$ & $0.012 *$ \\
\hline Peripheral location (\%) & $7(18.4)$ & $8(2 I . I)$ & 1.000 \\
\hline Site (\%) & & & 0.687 \\
\hline Unilateral & $16(42.1)$ & $14(36.8)$ & \\
\hline Bilateral & $22(57.9)$ & $24(63.2)$ & \\
\hline Microcalcification (\%) & $8(2 \mid . I)$ & $14(36.8)$ & 0.070 \\
\hline Multiple lesion (\%) & $24(63.2)$ & $28(73.7)$ & 0.219 \\
\hline Lymph node metastasis (\%) & $3(7.9)$ & $4(10.5)$ & 1.000 \\
\hline
\end{tabular}

Notes: Bold font: significant difference. $*_{p}<0.05$.

patients. Many medical institutions have had to adopt various measures to reduce nosocomial infections, including limiting patient admission or even lockdowns. ${ }^{28-30}$ These measurements, although effective in minimizing nosocomial infections, have inevitably led to diagnosis and treatment delays among cancer patients. Wuhan, which was the first centre of the pandemic, underwent a regional lockdown for 76 days. ${ }^{31}$ Many oncologists in Wuhan believe that thyroid cancers, although indolent in general, have shown more aggressive behaviour after the lockdown. There were some reviews or clinical experiences reported on thyroid cancer in the pandemic period, ${ }^{32,33}$ but clinical evidence is lacking.

Our study supports this hypothesis. Although demographic characteristics remained stable in general, thyroid cancer patients after the pandemic had a greater number of multifocal lesions, extrathyroidal extensions, and lymph node metastases on postoperative pathological examinations. Lymph node metastases have been widely recognized as risk factors for aggressiveness and metastasis. ${ }^{34}$ In addition, multifocality and extrathyroidal extension are powerful predictors for thyroid cancer. ${ }^{35,36}$ We may think with evidence that patients enduring a treatment delay will have a poorer prognosis in the future.

There is, obviously, more than one interpretation for the clinical findings. One may easily think that thyroid cancers have become more aggressive year by year. Our study showed that although tumour size and ETE remained almost unchanged, the incidence of LNM, especially central LNM, has increased by more than $12 \%$ in the past four years. These clinical findings may be explained by the increase in cervical lymph node ultrasounds and fineneedle aspiration biopsies being performed in clinical practice. Morris et al also found that the rate of cervical metastases has doubled over the past 40 years. ${ }^{21}$ Although evidence supports that increasing numbers of diagnostic tests leads to an increase in thyroid cancer incidence, ${ }^{37}$ we cannot abandon the hypothesis that the incidence has truly increased.

Rebound medical visits were another competing hypothesis. Rebound medical visits are defined as the increasing number of medical visits by worried patients after lockdown. Many thyroid cancer patients undergo surveillance before medical interventions. Those with more high-risk tumours may be more worried about their illness, and thus have been passionate to take on medical visits, which might have subsequently led to the observation of a pseudo-deterioration after the lockdown.

Table 5 Comparison of Ultrasound Characteristics in 19 PTMC Patients and 16 Patients with Treatment Delay $\leq 180$ Days

\begin{tabular}{|c|c|c|c|c|c|c|}
\hline \multirow[t]{2}{*}{ Variable } & \multicolumn{3}{|c|}{ PTMC } & \multicolumn{3}{|c|}{ Delayed Time $\leq 180 \mathrm{ds}$} \\
\hline & Pre-Lockdown & Post-Lockdown & $P$ value & Pre-Lockdown & Post-Lockdown & $P$ value \\
\hline Tumor size $(\mathrm{cm}, \mathrm{SD})$ & $0.74(0.36)$ & $0.70(0.25)$ & 0.357 & $1.00(1.08)$ & $\mathrm{I} .20(\mathrm{I} .44)$ & 0.092 \\
\hline Aspect ratio>I (\%) & $5(26.3)$ & $5(26.3)$ & 1.000 & $5(3 \mid .3)$ & $6(37.5)$ & 1.000 \\
\hline Irregular margin (\%) & $9(47.4)$ & $12(63.2)$ & 0.375 & $10(62.5)$ & II (68.8) & 1.000 \\
\hline Peripheral location (\%) & $2(10.5)$ & $3(15.8)$ & 1.000 & $3(18.8)$ & $4(25.0)$ & 1.000 \\
\hline Site (\%) & & & 1.000 & & & 1.000 \\
\hline Unilateral & $8(42.1)$ & $8(42.1)$ & & $8(50.0)$ & $7(43.8)$ & \\
\hline Bilateral & II (57.9) & II (57.9) & & $8(50.0)$ & $9(56.2)$ & \\
\hline Microcalcification (\%) & $4(2 \mid . I)$ & $4(2 \mid . I)$ & 1.000 & $4(25.0)$ & $5(3 \mid .3)$ & 1.000 \\
\hline Multiple lesion (\%) & $12(63.2)$ & $13(68.4)$ & 1.000 & $10(62.5)$ & $12(75.0)$ & 0.625 \\
\hline Lymph node metastasis (\%) & $0(0.0)$ & I (5.3) & $\mathrm{N} / \mathrm{C}$ & I (6.3) & $2(12.5)$ & 1.000 \\
\hline
\end{tabular}

Abbreviation: N/C, not calculable. 
Our analysis supported this theory. Pathological tumour size correlated negatively with the admission date. The average tumour size decreased at a speed of approximately $4 \mathrm{~mm}$ every 100 days from the first day of reopening. The research by Falcone et al supported that thyroid cancer patients were more anxious and distressed in the face of the pandemic. ${ }^{38}$ A shortcoming of this theory is that the rate of LNM remained stable after the reopening, which could not explain the difference between the two cohorts of patients.

The third hypothesis was that some thyroid carcinomas, especially those with higher risks, had progressed during the pandemic, similar to the progression of breast or lung cancer. ${ }^{39}$ Researchers thus pooled the patients with ultrasound records both before and after the lockdown for the analysis. Although the TI-RADS scores were somewhat higher in the postlockdown ultrasound group, other variables remained stable, indicating that most tumour remained indolent through the lockdown era. In the regression analysis, tumour size on prepandemic ultrasound was the only predictor for tumour enlargement and LNM over the lockdown period. The results were consistent with some investigations on active surveillance. ${ }^{40}$ These findings do not support the idea that those tumours progressed during the pandemic-induced lockdown.

In addition, the clinical management of thyroid cancer has faced challenges during the COVID-19 pandemic. Scholars noticed that the delay time has had almost no effect on patient outcomes. Subsequent analyses clarified that thyroid cancer patients can endure a period of treatment delay up to 180 days. Moreover, our study shows that the tumour size and rate of LNM remained stable after lockdown in patients with tumours no more than $10 \mathrm{~mm}$. The comparison among the total patient sample before and after COVID-19 also supports this theory. Some guidelines have recommended cervical ultrasound, thyroid scintigraphy, and fine-needle biopsy as the main methods in decision-making. ${ }^{25,41}$ Due to the pandemic and the subsequent lockdown, patients are now advised to take teleconsultations or to postpone their surgery. Low-to-intermediate-risk patients can tolerate a 180-day delay without tumour progression, a conclusion that is supported by clinical experience. ${ }^{42}$

There were some limitations to our study. First, the patient number in the pre- and post-pandemic ultrasound comparison was too short to draw more solid conclusions. In addition, although the 3253 patients included had either pathology or ultrasound records (most patients had both), a minority of patients had missing items, which may have led to information bias. This was a single-centre retrospective study, and the generalization of our theory is thus limited. Moreover, some pathological characteristics, including subcapsular or isthmic nodule location, were missing in our pathological records. Campennì et al revealed that isthmus topography is an important predictive factor for persistent disease and metastasis. ${ }^{43,44}$ Further prospective studies with larger sample sizes from multiple centres are needed to evaluate our results.

\section{Conclusion}

In conclusion, our study revealed more aggressive thyroid cancer behaviour in patients after a long period of COVID-19 induced treatment delay. However, as most tumours were indolent, they did not progress during the lockdown period. The more high-risk signs in postoperative pathology can thus be interpreted as due to rebound medical visits from patients' anxiety, as well as to an annual increase in tumour aggressiveness. In addition, our study revealed that thyroid cancer patients with tumours less than $10 \mathrm{~mm}$ in diameter could tolerate a long treatment delay without any notable progression, and a delay of no more than 180 days is tolerable for most patients. These findings will help clinicians better evaluate thyroid cancer patients in the era of pandemics.

\section{Data Sharing Statement}

All data generated or analyzed during this study are available from the corresponding author on reasonable requests.

\section{Acknowledgments}

The authors thank all patients who provided their data in the analysis.

\section{Author Contributions}

All authors contributed to data analysis, drafting or revising the article, gave final approval of the version to be published, agreed to the submitted journal, and agree to be accountable for all aspects of the work.

\section{Funding}

This work was supported by the National Natural Science Foundation of China [grant number 81471781], the Key Talent Training Program of Renmin Hospital and the Foundation for the Construction of National Medical and Health Service Center, and the Fundamental Research Funds for the Central Universities [grant number 2042019kf0229]. 


\section{Disclosure}

Dr Chuang Chen reports grants from Fundamental Research Funds for the Central Universities, grants from Key Talent Training Program of Renmin Hospital, grants from Foundation for the Construction of National Medical and Health Service Center, during the conduct of the study. Prof. Dr. Shengrong Sun reports grants from National Natural Science Foundation of China, during the conduct of the study. The authors have declared no conflicts of interest.

\section{References}

1. Coronavirus disease (COVID-19) pandemic [homepage on the Internet]. Geneva: World Health Organization; 2021. Available from: https://www.who.int/emergencies/diseases/novel-coronavirus -2019. Accessed September 17, 2021.

2. Klompas M, Morris CA, Sinclair J, Pearson M, Shenoy ES. Universal masking in hospitals in the Covid-19 era. $N$ Engl J Med. 2020;382 (21):e63. doi:10.1056/NEJMp2006372

3. Lee JK, Jeong HW. Wearing face masks regardless of symptoms is crucial for preventing the spread of COVID-19 in hospitals. Infect Control Hosp Epidemiol. 2020;42(1):115-116. doi:10.1017/ ice. 2020.202

4. Bhatla N, Singhal S. The COVID-19 pandemic and implications for gynaecologic cancer care. Indian J Gynecol Oncol. 2020;18(2):48. doi:10.1007/s40944-020-00395-7

5. Mlcochova P, Collier D, Ritchie A, et al. Combined point-of-care nucleic acid and antibody testing for SARS-CoV-2 following emergence of D614G spike variant. Cell Rep Med. 2020;1(6):100099. doi:10.1016/j.xcrm.2020.100099

6. Frey MK, Fowlkes RK, Badiner NM, et al. Gynecologic oncology care during the COVID-19 pandemic at three affiliated New York City hospitals. Gynecol Oncol. 2020;159(2):470-475. doi:10.1016/j. ygyno.2020.09.005

7. Papautsky EL, Hamlish T. Patient-reported treatment delays in breast cancer care during the COVID-19 pandemic. Breast Cancer Res Treat. 2020;184(1):249-254. doi:10.1007/s10549-020-05828-7

8. Sha Z, Chang K, Mi J, et al. The impact of the COVID-19 pandemic on lung cancer patients. Ann Palliat Med. 2020;9(5):3373-3378. doi:10.21037/apm-20-1662

9. Li J, Santa-Maria CA, Feng H, et al. Patient-reported outcomes of patients with breast cancer during the COVID-19 outbreak in the epicenter of China: a cross-sectional survey study. Clin Breast Cancer. 2020;20(5):E651-E662. doi:10.1016/j.clbc.2020.06.003

10. Casco NC, Carmona MJ, Soto AJ. Therapeutic and surgical indications for patients with penile cancer in the COVID-19 era. Int Braz J Urol. 2020;46(suppl.1):86-92. doi:10.1590/s1677-5538.ibju.2020. s110

11. Shipe ME, Haddad DN, Deppen SA, Kozower BD, Grogan EL. Modeling the impact of delaying the diagnosis of non-small cell lung cancer during COVID-19. Ann Thorac Surg. 2020;19:248-254.

12. Lung Cancer Study Group CTSCMA, Chinese Respiratory Oncology C. Expert recommendations on the management of patients with advanced non-small cell lung cancer during epidemic of coronavirus disease 2019 (Trial version). Zhonghua Jie He He Hu Xi Za Zhi. 2020;43(4):297-301.

13. Brissot E, Labopin M, Baron F, et al. Management of patients with acute leukemia during the COVID-19 outbreak: practical guidelines from the acute leukemia working party of the European Society for Blood and Marrow Transplantation. Bone Marrow Transplant. 2021;56(3):532-535. doi:10.1038/s41409-020-0970-x
14. Conforti C, Giuffrida R, Di Meo N, Zalaudek I. Management of advanced melanoma in the COVID-19 era. Dermatol Ther. 2020;33 (4):e13444. doi:10.1111/dth.13444

15. Raghavan D, Tan AR, Story ES, et al. Management changes for patients with endocrine-related cancers in the COVID-19 pandemic. Endocr Relat Cancer. 2020;27(9):E357-E374. doi:10.1530/ERC-20-0229

16. Assi T, Ibrahim N, Abboud RMK, et al. The management of patients with metastatic prostate cancer during the COVID-19 pandemic. Fut Oncol. 2020;16(20):1455-1462. doi:10.2217/fon-2020-0361

17. Salazar-Vega J, Ortiz-Prado E, Solis-Pazmino P, et al. Thyroid cancer in Ecuador, a 16 years population-based analysis (2001-2016). BMC Cancer. 2019;19(1):294. doi:10.1186/s12885-019-5485-8

18. Hoang JK, Nguyen XV, Davies L. Overdiagnosis of thyroid cancer: answers to five key questions. Acad Radiol. 2015;22(8):1024-1029. doi:10.1016/j.acra.2015.01.019

19. O'Grady TJ, Gates MA, Boscoe FP. Thyroid cancer incidence attributable to overdiagnosis in the United States 1981-2011. Int J Cancer. 2015;137(11):2664-2673. doi:10.1002/ijc.29634

20. Davies L, Hoang JK. Thyroid cancer in the USA: current trends and outstanding questions. Lancet Diabetes Endocrinol. 2021;9(1):11-12. doi:10.1016/S2213-8587(20)30372-7

21. Morris LGT, Myssiorek D. Improved detection does not fully explain the rising incidence of well-differentiated thyroid cancer: a population-based analysis. Am J Surg. 2010;200(4):454-461. doi:10.1016/j.amjsurg.2009.11.008

22. Bresner L, Banach R, Rodin G, Thabane L, Ezzat S, Sawka AM. Cancer-related worry in Canadian thyroid cancer survivors. J Clin Endocrinol Metab. 2015;100(3):977-985. doi:10.1210/jc.2014-3169

23. Andersen JH, Jensen JC. Modern health worries and visits to the general practitioner in a general population sample: an 18 month follow-up study. $J$ Psychosom Res. 2012;73(4):264-267. doi:10.1016/j.jpsychores.2012.07.007

24. Fenton JJ, Magnan EM, Jerant A, Kravitz RL, Franks P. Patient characteristics associated with making requests during primary care visits. J Am Board Fam Med. 2019;32(2):201-208. doi:10.3122/ jabfm.2019.02.180218

25. Campenni A, Barbaro D, Guzzo M, Capoccetti F, Giovanella L. Personalized management of differentiated thyroid cancer in real life - practical guidance from a multidisciplinary panel of experts. Endocrine. 2020;70(2):280-291. doi:10.1007/s12020-020-02418-x

26. Health Commission of Hubei Province. In extraordinary times, necessary diagnosis and treatment are guaranteed, Wuhan medical institutions are in action [homepage on the Internet]. Wuhan: Health Commission of Hubei Province; 2021. Available from: http://wjw. hubei.gov.cn/bmdt/ztzl/fkxxgzbdgrfyyq/fkdt/202002/t20200214 2027302.shtml. Accessed February 14, 2021.

27. Tessler FN, Middleton WD, Grant EG, et al. ACR Thyroid Imaging, Reporting and Data System (TI-RADS): white paper of the ACR TI-RADS committee. $J$ Am Coll Radiol. 2017;14(5):587-595. doi:10.1016/j.jacr.2017.01.046

28. Tsang VHM, Gild M, Glover A, Clifton-Bligh R, Robinson BG. Thyroid cancer in the age of COVID-19. Endocr Relat Cancer. 2020;27(11):R407-R416. doi:10.1530/ERC-20-0279

29. Krishan K, Kanchan T. Lockdown is an effective 'vaccine' against COVID-19: a message from India. J Infect Dev Ctries. 2020;14 (6):545-546. doi:10.3855/jidc. 12931

30. Siqueira CA, de Freitas YNL, Cancela MD, Carvalho M, OliverasFabregas A, de Souza DLB. The effect of lockdown on the outcomes of COVID-19 in Spain: an ecological study. PLoS One. 2020;15(7): e0236779. doi:10.1371/journal.pone.0236779

31. Qian Y, Hanser A. How did Wuhan residents cope with a 76-day lockdown? Chin Sociol Rev. 2020;53(1):55-86. doi:10.1080/ 21620555.2020.1820319

32. Smulever A, Abelleira E, Bueno F, Pitoia F. Thyroid cancer in the Era of COVID-19. Endocrine. 2020;70(1):1-5. doi:10.1007/s12020020-02439-6 
33. Vrachimis A, Iakovou I, Giannoula E, Giovanella L. Endocrinology in the time of COVID-19 management of thyroid nodules and cancer. Eur J Endocrinol. 2020;183(1):G41-G48. doi:10.1530/EJE-20-0269

34. Haugen BR, Alexander EK, Bible KC, et al. 2015 American Thyroid Association management guidelines for adult patients with thyroid nodules and differentiated thyroid cancer The American Thyroid Association guidelines task force on thyroid nodules and differentiated thyroid cancer. Thyroid. 2016;26(1):1-133.

35. Joseph KR, Edirimanne S, Eslick GD. Multifocality as a prognostic factor in thyroid cancer: a meta-analysis. Int $J$ Surg. 2018;50:121-125. doi:10.1016/j.ijsu.2017.12.035

36. Park YM, Lee DY, Oh KH, et al. Clinical implications of pathologic factors after thyroid lobectomy in patients with papillary thyroid carcinoma. Oral Oncol. 2017;75:1-5. doi:10.1016/j. oraloncology.2017.10.012

37. Olson E, Wintheiser G, Wolfe KM, Droessler J, Silberstein PT. Epidemiology of thyroid cancer: a review of the national cancer database, 2000-2013. Cureus. 2019;11(2):e4127.

38. Falcone R, Grani G, Ramundo V, et al. Cancer care during COVID-19 era: the quality of life of patients with thyroid malignancies. Front Oncol. 2020;10:1128. doi:10.3389/ fonc. 2020.01128

39. Akula SM, Abrams SL, Steelman LS, et al. Cancer therapy and treatments during COVID-19 era. Adv Biol Regul. 2020;77:100739. doi:10.1016/j.jbior.2020.100739
40. Kim TY, Shong YK. Active surveillance of papillary thyroid microcarcinoma: a mini-review from Korea. Endocrinol Metab. 2017;32 (4):399-406. doi:10.3803/EnM.2017.32.4.399

41. Tuttle RM, Ahuja S, Avram AM, et al. Controversies, consensus, and collaboration in the use of I-131 therapy in differentiated thyroid cancer: a joint statement from the American Thyroid Association, the European Association of Nuclear Medicine, the Society of Nuclear Medicine and Molecular Imaging, and the European Thyroid Association. Thyroid. 2019;29(4):461-470.

42. Shaha AR. Thyroid surgery during COVID-19 pandemic: principles and philosophies. Head Neck. 2020;42(6):1322-1324. doi:10.1002/ hed. 26198

43. Campenni A, Giovanella L, Siracusa M, et al. Is malignant nodule topography an additional risk factor for metastatic disease in low-risk differentiated thyroid cancer? Thyroid. 2014;24(11):1607-1611. doi:10.1089/thy.2014.0217

44. Campenni A, Ruggeri RM, Siracusa M, et al. Isthmus topography is a risk factor for persistent disease in patients with differentiated thyroid cancer. Eur $J$ Endocrinol. 2021;185(3):397-404. doi:10.1530/EJE-21-0328
International Journal of General Medicine

\section{Publish your work in this journal}

The International Journal of General Medicine is an international, peer-reviewed open-access journal that focuses on general and internal medicine, pathogenesis, epidemiology, diagnosis, monitoring and treatment protocols. The journal is characterized by the rapid reporting of reviews, original research and clinical studies

\section{Dovepress}

across all disease areas. The manuscript management system is completely online and includes a very quick and fair peer-review system, which is all easy to use. Visit http://www.dovepress.com/ testimonials.php to read real quotes from published authors. 